\title{
PATZ1 downregulation promotes proliferation and migration in Ras-driven thyroid transformation
}

\author{
Michela Vitiello ${ }^{1}$, Teresa Valentino ${ }^{1}$, Marta de Menna ${ }^{2}$, Paola Francesca ${ }^{1}$, Gabriella de Vita ${ }^{2}$ \\ and Monica Fedele 1
}

${ }^{1}$ Istituto per l'Endocrinologia e l'Oncologia Sperimentale (IEOS) - CNR, 80131 Naples, Italy.

${ }^{2}$ Dipartimento di Medicina Molecolare e Biotecnologie Mediche, Università degli studi di Napoli Federico II, Italy

\section{Introduction and Objectives}

Carcinoma of the thyroid gland is one of the most frequent malignancies of the endocrine system and its incidence has been steadily increased in many regions of the world. Among the most frequent genetic alterations occurring in thyroid transformation, oncogenic mutations of RASfamily genes have been identified in all types of thyroid malignancies, and are considered to be both an early and late event in thyroid tumorigenesis. Indeed, the expression of the oncogenic Ras $V 12$, induced by tamoxifen in FRTL5 rat thyroid cells, caused complete malignant transformation towards an undifferentiated phenotype, confirming that Ras could contribute to the partial or complete loss of differentiation, characteristic of the more aggressive thyroid cancers (1). Oncogenic Ras is able to induce aberrant expression of miRNAs during the transformation of thyroid epithelial cells (2). Among the up-regulated miRNAs there is miR-29b, a miRNA that we showed to directly target PATZ1, a protein belonging to the POZ and Zinc finger family of transcriptional factors. Recently, we found that PATZ1 is downregulated in thyroid cancer and acts as a tumor suppressor in human thyroid cancer cells mainly by inhibiting cell migration and partially reverting the Epithelial-Mesenchimal Transition (3). Subsequently, we found that it is specifically downregulated downstream of oncogenic Ras during thyroid transformation, with an inverse correlation with miR-29b expression (see figure on the side), thus suggesting a new RasmiR-29b-PATZ1 axis in thyroid carcinogenesis. Here, we investigated whether the downregulation of PATZ1 plays a causal role in Ras-induced thyroid transformation. Elucidation of the molecular pathways downstream of oncogenic Ras, which are crucial for thyroid transformation, will help to find new therapeutic targets in thyroid cancer carrying Ras mutations.

\section{Results}

1. Restoration of PATZ1 expression in FRTL5-Ras cells

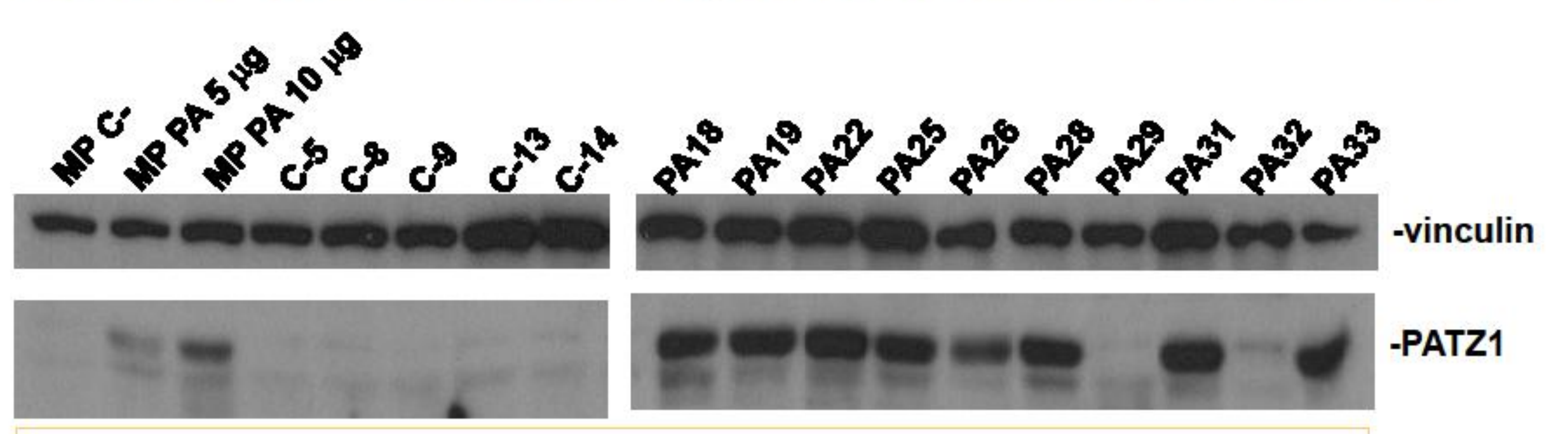

Western Blot analysis of PATZ1 in cell clones and mass populations (MP) obtained by transfection of either a PATZ1expressing plasmid (PA) or the empty vector (C-). Vinculin expression has been analyzed as a loading control.

2. PATZ1 expression in Ras-transformed thyroid cells inhibits proliferation

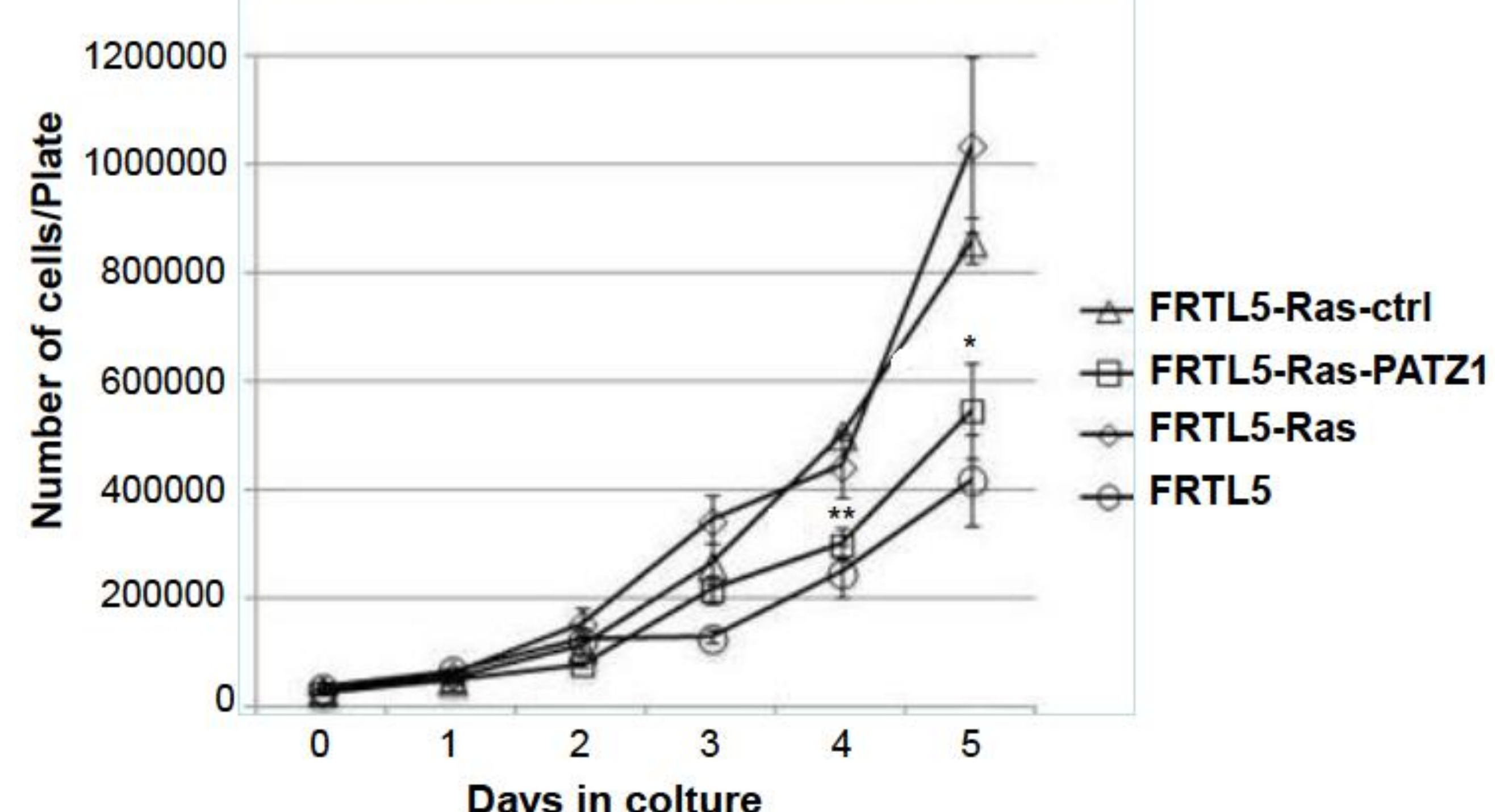

Growth curves (mean values \pm SE of three different clones) Ras) and normal control (FRTL5) cells. ${ }^{*}, \mathrm{P}<0.05 ;{ }^{* *}, \mathrm{P}<0.01$, as assessed by unpaired T test. performed on PATZ1-expressing (FRTL5-Ras-PATZ1) compared to empty vector-transfected (FRTL5-Ras-ctrl), parental (FRTL-

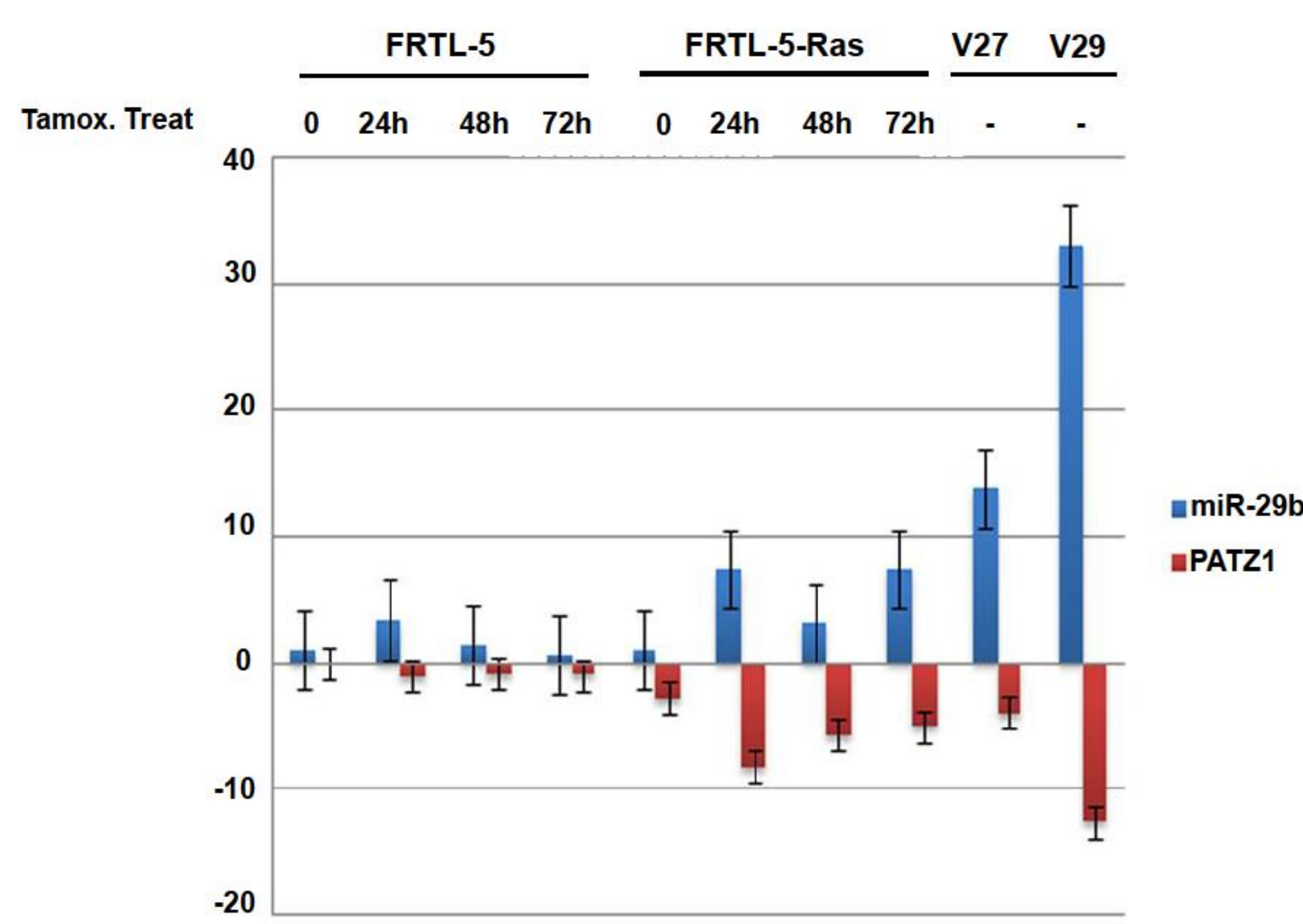

qRT-PCR analysis showing miR-29b up-regulation following induction of Ras as early as after $24 \mathrm{~h}$ of treatment with tamoxifen and in two FRTL-5 cell clones stably expressing an oncogenic Ras (V27 and V29). Opposed to miR-29b, PATZ1 resulted strongly downregulated. Data represent mean values \pm SE of three independent experiments performed in triplicate.

4. PATZ1 expression in Ras-transformed thyroid cells inhibits transwell migration
3. PATZ1 expression in Ras-transformed thyroid cells inhibits wound closure
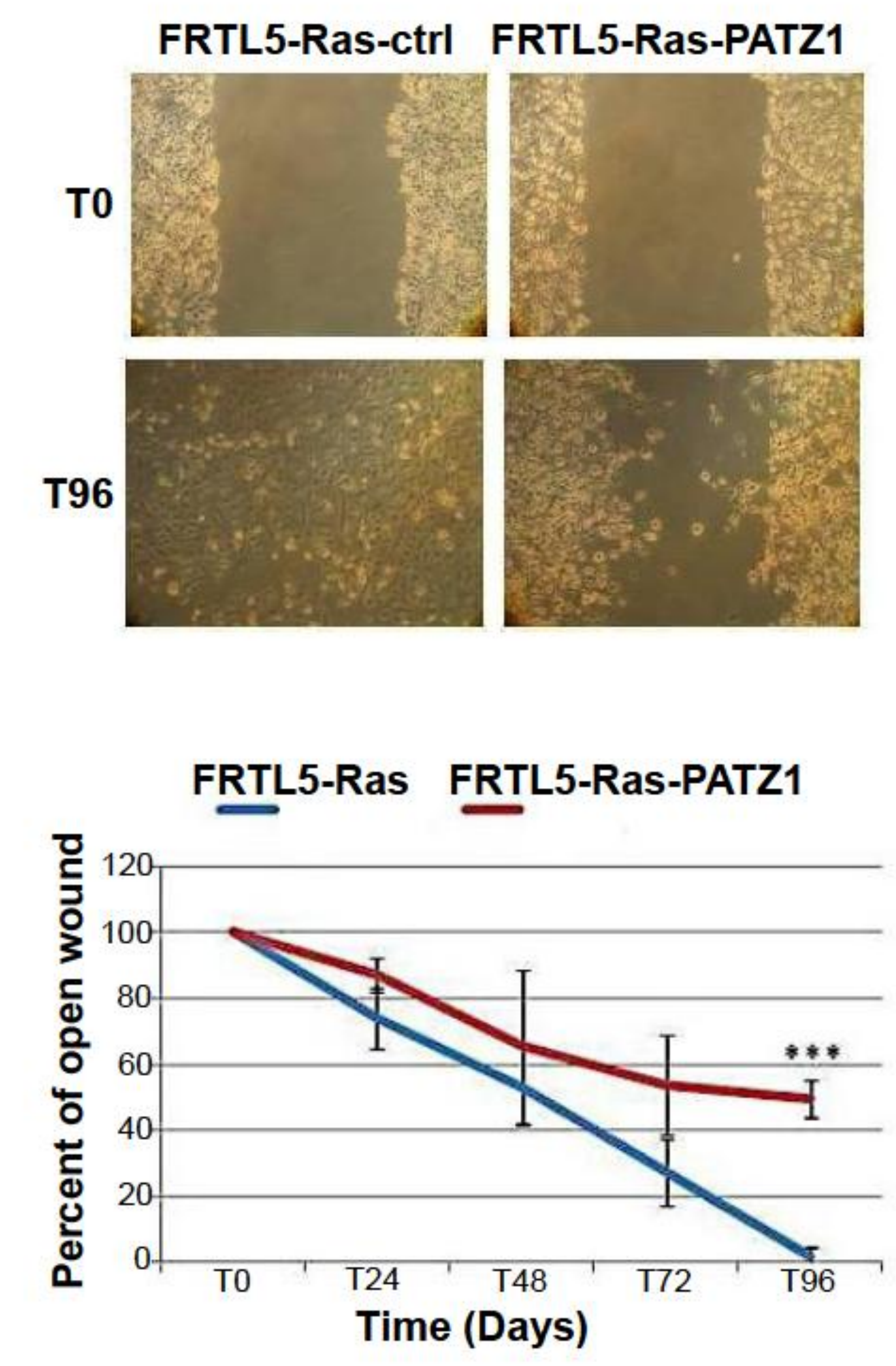

Representative images of one control clone expressing the empty vector and one clone overexpressing PATZ1. The graph shows the percent of open wound at different time points expressed as mean values \pm SE of three control clones and three clones overexpressing PATZ1. ${ }^{* * *}, \mathrm{P}<0.001$, as assessed by unpaired T test.

\section{Conclusions}

* In Ras ${ }^{12}$-expressing FRTL5 cells PATZ1 is downregulated.

* Expression of miR-29b, which targets PATZ1, is inversely correlated to PATZ1 during Ras-induced thyroid transformation in FRTL5 cells

* PATZ1 transfection in Ras ${ }^{\mathrm{V} 2}$-expressing FRTL5 cells is able to partially revert their transformed phenotype in terms of:

$>$ proliferation

$>$ migration;

$>$ tumorigenicity

All in all these results suggest that PATZ1 is a pivotal regulator acting downstream of Ras (likely via miR-29b) to suppress thyroid cell transformation driven by oncogenic Ras, highlighting a new potential therapeutic target to fight highly malignant thyroid cancer.

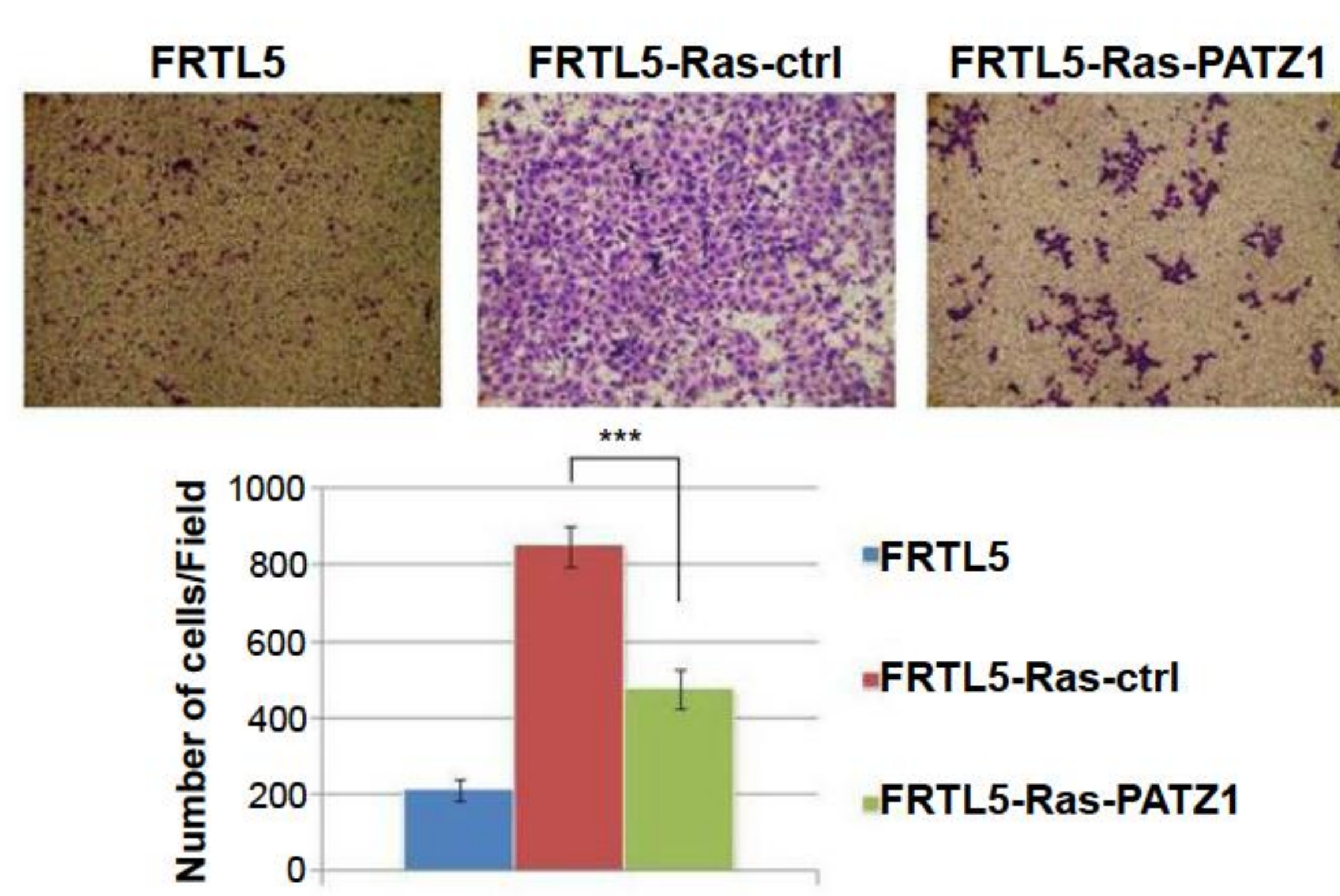

Representative images of a Transwell assay performed on FRTL5, FRTL5-Ras-ctrl and FRTL5-Ras-PATZ1 cells. The graph shows the mean values + SE of three clones for each cells that have migrated underneath the Boyden chamber. ***, $\mathrm{P}<0.001$ as assessed by Unpaired T test.

5. PATZ1 expression in Ras-transformed thyroid cells inhibits tumorigenicity

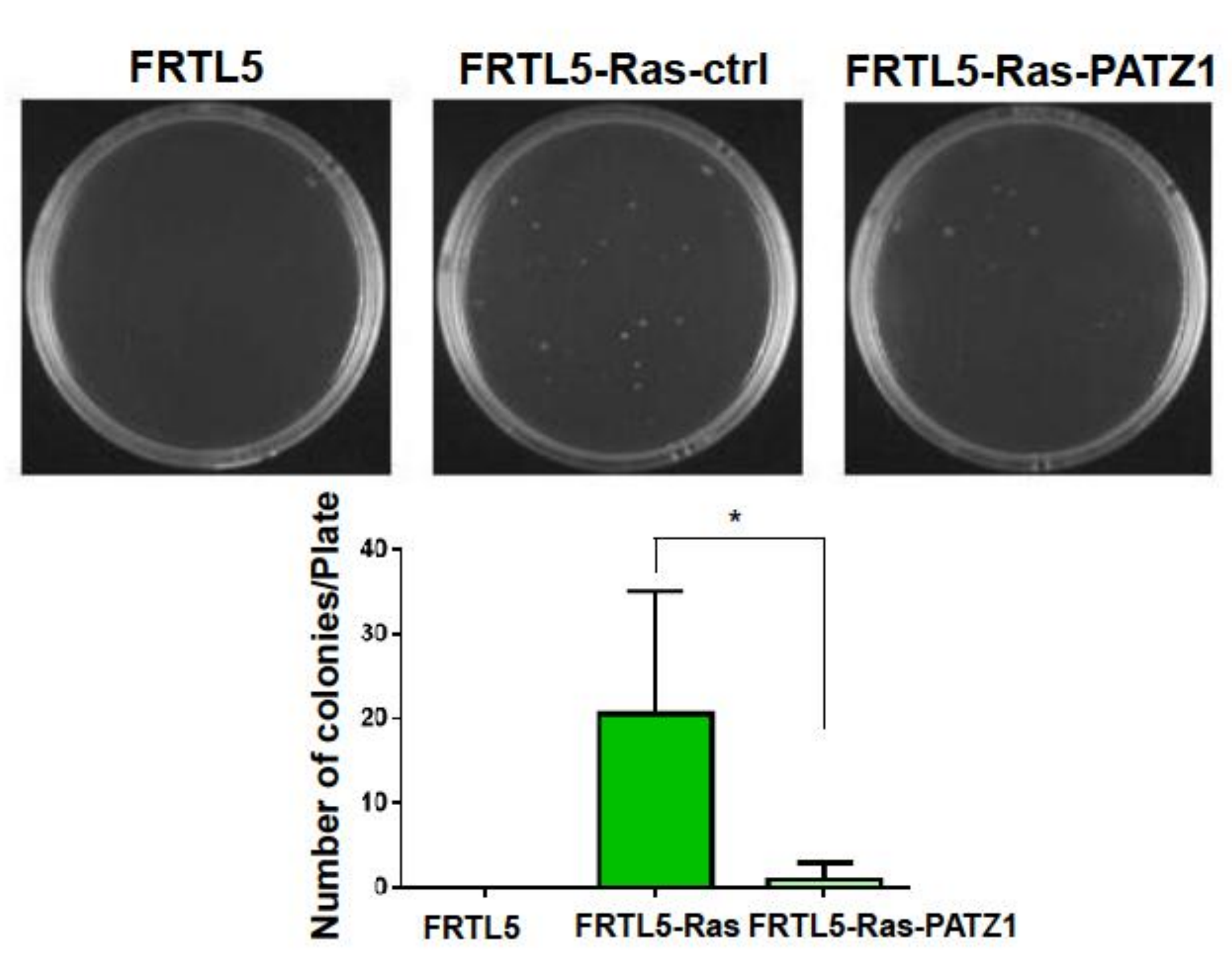

Representative images of growth in soft agar of normal rat FRTL5, FRTL5-Ras-ctrl and FRTL5-Ras-PATZ1 cells The graph shows the mean values \pm SE of the colony number in four plates for each cell line * $\mathrm{P}<0.05$, as assessed by Unpaired T test.

\section{References:}

. De Vita G, et al. Dose-dependent inhibition of thyroid differentiation by RAS oncogenes. Mol Endocrinol. 2005;19:76-89.

Landgraf $\mathrm{P}$, et al. A mammalian microRNA expression atlas based on small RNA library sequencing. Cell 2007;129:1401-14.

Chiappetta G, Valentino T et al. PATZ1 acts as a tumor suppressor in thyroid cancer via targeting p53-dependent genes involved in EMT and cell migration. Oncotarget 2015; 6:5310-23. transfected construct obtained by counting the number of 